\title{
A primary care database study of asthma among patients with and without opioid use disorders
}

\author{
Phillip Oliver $\mathbb{D}^{1 凶}$, Joe Hulin $\mathbb{D}^{2}$ and Caroline Mitchell $\mathbb{D}^{1}$
}

Substance misuse is associated with poor asthma outcome and death. People with opioid use disorder (OUD) may be at particular risk, however, there have been no case-control studies of asthma care and outcomes in this patient group. A primary care database study of patients with asthma aged 16-65 years was conducted using a matched case-control methodology. The dataset comprised 275,151 adults with asthma, of whom 459 had a clinical code indicating a lifetime history of OUD. Cases with a history of OUD were matched to controls $1: 3$ by age, gender, smoking status and deprivation index decile. Attendance at annual review (30\%) and for immunisation $(25 \%)$ was poor amongst the overall matched study population $(N=1832)$. Compared to matched controls, cases were less likely to have attended for asthma review during the previous 12 months $(\mathrm{OR}=0.60,95 \% \mathrm{Cl} 0.45-0.80)$ but had similar immunisation rates. Higher rates of ICS $(O R=1.50,1.13-1.98)$ and oral prednisolone use $(O R=1.71,1.25-2.40)$ were seen amongst those with a history of OUD and $7.2 \%$ had a concurrent diagnosis of COPD (OR=1.86, 1.12-2.40). We found that people with asthma and a history of OUD have worse outcomes on several commonly measured metrics of asthma care. Further research is required to identify reasons for these findings, the most effective strategies to help this vulnerable group access basic asthma care, and to better understand long-term respiratory outcomes.

npj Primary Care Respiratory Medicine (2020)30:17; https://doi.org/10.1038/s41533-020-0174-2

\section{INTRODUCTION}

Asthma is amongst the most common long-term health conditions in the UK ${ }^{1}$ and represents a significant burden for patients, general practice, emergency medicine and secondary care. Small scale observational studies suggest that people with asthma and opioid use disorder (OUD) experience high rates of acute exacerbations $^{2}$, have more severe symptoms during acute episodes $s^{3,4}$ and are more likely to need intensive care treatment and intubation ${ }^{5,6}$. The UK has one of the highest rates of death from asthma in Europe $^{7}$ and in the 2014 UK National Review of Asthma Deaths, substance misuse was identified as a factor in $6 \%$ of the cases ${ }^{8}$. Supported self-management such as medication adherence ${ }^{9}$, attendance at asthma review ${ }^{10,11}$ and immunisation ${ }^{12}$ are known to improve outcome. However, people with substance use disorders (SUD) often live with significant psychosocial challenges, for example poverty, stigma, family breakdown, insecure housing and incarceration, which may affect their access to healthcare services ${ }^{13}$.

Lack of healthcare utilisation may explain why outcomes amongst people with asthma and SUD appear to be so poor, but the nature of specific illicit drug use might also be a significant additional factor for those with OUD. As a part of the harm minimisation paradigm, injecting heroin users were encouraged to adopt alternative routes of drug use and by the late 1990s, inhalation became a common method of administration in many countries $^{14,15}$. These 'route transition interventions' were aimed at reducing the risk of fatal overdose, blood-borne virus transmission and intravenous drug injection-associated infections. However, inhalation of heroin has bronchoconstrictive effects in the lungs ${ }^{3}$, probably mediated by the ability of morphine to cause histamine release $^{16}$, and appears to contribute to progressive irreversible lung disease, such as emphysema and chronic obstructive pulmonary disease $(\mathrm{COPD})^{17,18}$. Assessing the contribution of inhalation of opioids to lung outcomes is challenging as the vast majority of those with SUD smoke tobacco ${ }^{19}$. It is known that people with SUD have significantly higher excess mortality than the general population from causes, including cancer, cardiovascular and respiratory disease $\mathrm{e}^{20}$, and it is likely that a significant proportion of this excess mortality is explained by tobacco smoking.

At present, there is little existing research that has attempted to quantify asthma outcomes among people with OUD. The majority of existing research comes from small scale and case-series data ${ }^{17}$. More generally, it is recognised that the literature on the pulmonary consequences of heroin use is scant ${ }^{18}$. When assessing asthma outcomes amongst people with OUD, it is important to acknowledge this group are more likely to be male, come from areas of high deprivation and have smoking histories ${ }^{5}$. Factors such as these have been shown to influence asthma outcomes including exacerbation rates ${ }^{21-24}$. This study, aims to assess asthma care and outcomes amongst asthma patients with a history of OUD in a large primary care population after controlling for such confounding.

\section{RESULTS}

Rates of OUD

In all, 275,151 patients aged 16-65 years with asthma were identified from the ResearchOne database. Of these, 459 had a clinical code indicating past or current OUD. Of the total number of patients, 254,568 had complete data available for matching (Fig. 1). There was no difference in the frequency of historical OUD between those with complete and those with missing data (Pearson $X^{2}=1.346, p=0.246$ ).

\footnotetext{
${ }^{1}$ Academic Unit of Primary Medical Care, University of Sheffield, Sheffield, UK. ${ }^{2}$ School of Health and Related Research, University of Sheffield, Sheffield, UK.

$\bigotimes_{\text {email: p.oliver@sheffield.ac.uk }}$
} 
Study population characteristics-matched groups

Comparison between the groups with respect to demographics and smoking history are shown in Table 1 along with the results of significance tests. As expected, statistically significant differences with respect to the measured confounding factors were seen. Whilst asthma patients with a history of OUD were only slightly older, they were much more likely to be male and considerably more likely to live in an area with the highest level of deprivation. Ninety five percent of those with a history of OUD had a history of smoking compared to $52 \%$ of non-opioid users.

\section{Outcomes-descriptive statistics}

After matching, the analysis population comprised 458 asthma patients with a history of past or current OUD (cases) and 1374 without (controls). Table 2 gives values and percentages for each of the outcome variables. Less than one third of the study sample had attended for an asthma review in the previous 12 months,

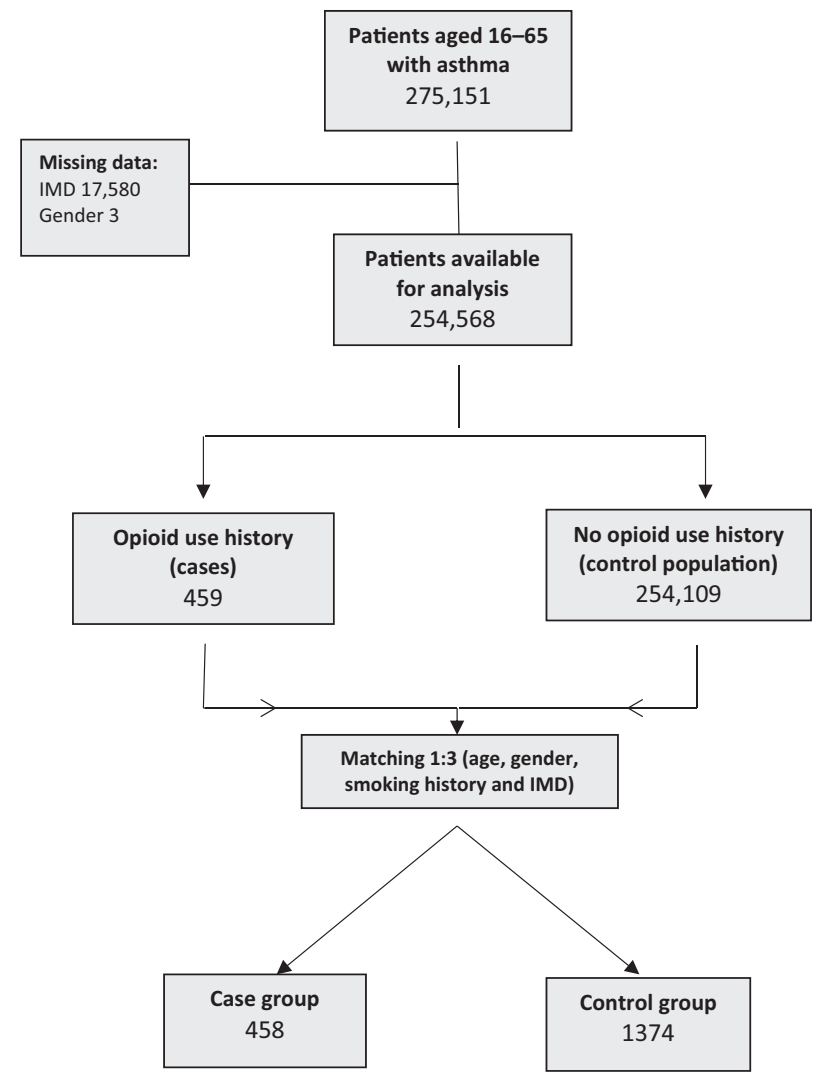

Fig. 1 Matched case-control methodology. Flow diagram showing process by which case and control groups were derived from the dataset. IMD index of multiple deprivation. with those in the control group more likely to have attended than cases ( $31 \%$ vs. $26 \%)$. More than three quarters of all patients had not received an influenza vaccination during the observation period with marginally higher rates of uptake amongst the control group ( $25 \%$ vs. $23 \%$ ). Asthma patients from the control group were less likely to have been issued three or more corticosteroid containing inhalers ( $29 \%$ vs. $36 \%$ ) in the previous 12 months with overall rates for both groups of $31 \%$. Approximately $8 \%$ of the 1832 patients received a prescription for oral prednisolone on at least one occasion in the 12-month period with cases recorded as having double the rates of controls (12 vs. 6\%). The overall prevalence of COPD amongst the study group was $4.5 \%$ with higher rates recorded for those with a history of OUD compared to controls (7.2\% vs. $3.6 \%)$.

Outcomes-odds ratios estimated from conditional logistic regression

Crude and adjusted odds ratios (ORs) with 95\% confidence intervals (Cls) for all outcome variables are shown in Table 3. Statistically significant ORs were found for all outcomes other than receiving an influenza vaccination $(95 \% \mathrm{Cl}$ 0.68-1.14). The adjusted odds of attending for an asthma review were around $40 \%$ lower for cases than controls $(95 \% \mathrm{Cl} 0.45-0.80)$ whereas the adjusted odds of receiving three or more ICS inhalers $(95 \% \mathrm{CI} 1.13$ to 2.98$)$, being issued a prescription for oral prednisolone $(95 \% \mathrm{Cl}$ $1.25-2.34)$, or having a pre-existing diagnosis of COPD $(95 \% \mathrm{Cl}$ $1.12-3.01$ ) were increased by $50 \%, 71 \%$ and $86 \%$ respectively. Crude and adjusted odds ratios were similar suggesting that there were no significant interactions between the outcome variables other than for co-existing COPD in which the adjusted OR fell from 2.26 to 1.86 .

\section{DISCUSSION}

Our findings confirm that asthma patients with evidence of previous OUD are more likely to be older, male, come from areas of higher deprivation and have a smoking history. Ignoring, for a moment the role of OUD, we found low rates of access to asthma care and inhaled ICS prescriptions amongst both cases and controls. In matched analyses, opioid users were less likely to attend for annual review but had higher rates of ICS prescriptions. Asthma patients with a history of OUD use had higher rates of oral prednisolone use within the previous 12 months and were more likely to have a co-existing clinical code for COPD. The latter was the only variable, which appeared to be influenced by other outcomes in multi-variable analyses.

Supported self-management forms the cornerstone of asthma treatment and has been shown to reduce hospital admission and unscheduled clinical contacts ${ }^{25}$. It was therefore, striking to find such low rates of achievement of the quality indicators for asthma care in this study. Less than one third of the 1832 patients had attended for an asthma review in the past 12 months and less

Table 1. Study population characteristics.

\begin{tabular}{llll}
\hline & History of opioid use & No history of opioid use & Significance tests \\
\hline Total (\%) & $459(0.18 \%)$ & $254,109(99.82 \%)$ & Mean difference $(95 \% \mathrm{Cl})$ \\
Mean age (SD) & 39.9 years (8.4) & 38.4 years (13.8) & 1.53 years $(0.27-2.8), p=0.018^{\mathrm{a}}$ \\
$\%$ Female & 38.8 & 51.3 & $x^{2}=28.16, p<0.001$ \\
$\%$ Smoking history & 94.8 & 52.0 & $x^{2}=335.83, p<0.001$ \\
$\%$ in most deprived IMD centile & 37.5 & 10.9 & $x^{2}=330.50, p<0.001$ \\
\hline Independent groups $t$-test, two-tailed $p$ value. & & \\
\hline
\end{tabular}


than a quarter had received an influenza vaccination. This is despite these aspects of asthma care being incentivised in England through the Quality Outcomes Framework (QoF) and the Enhanced Services Specification ${ }^{26}$, respectively. The rates of attendance an asthma review in our matched sample were considerably lower than the rates among the UK general population of asthma patients in similar database. For example, in a recent study using the Clinical Practice Research Datalink (CPRD) (an alternative UK GP database to ResearchOne), $43.7 \%$ of patients had attended for asthma review ${ }^{27}$. After controlling for measured confounding and other outcomes variables, we found that OUD was associated with a $40 \%$ reduction in the odds of having attended for asthma review. The finding of higher rates of ICS prescriptions among those with a history of OUD is somewhat at odds with this and has several possible interpretations. Assuming that both groups have similar severity of asthma requiring regular ICS-preventer therapy, then, taken at face value, this suggests higher adherence amongst those with a history of OUD. In the context of other chronic health conditions such as HIV medication adherence is known to be lower in those with SUD ${ }^{28}$ and so this would seem unlikely. An alternative explanation is that clinical need for an ICS-containing inhaler was different between the two groups and the higher OR is a reflection of this rather than differences in adherence per se. This would be consistent with the notion that OUD is associated with poorer lung function in those with asthma. It is also known that use of ICS increases during the period before and after exacerbations ${ }^{29}$, which could also explain the direction of this outcome. A national sample of data from the CPRD reported by Bloom et al., indicate that around $65 \%$ of people with asthma aged $18-54$ years received $\geq 3$ ICS inhalers in

\begin{tabular}{|c|c|c|c|}
\hline \multirow[t]{2}{*}{$\begin{array}{l}\text { Outcome (past } \\
12 \text { months) }\end{array}$} & $\begin{array}{l}\text { History of } \\
\text { opioid use } \\
\text { (cases) } \\
n=458\end{array}$ & $\begin{array}{l}\text { No history of } \\
\text { opioid use } \\
\text { (controls) } \\
n=1374\end{array}$ & $\begin{array}{l}\text { All } \\
N=1832\end{array}$ \\
\hline & $\begin{array}{l}\text { Number } \\
\%\end{array}$ & $\begin{array}{l}\text { Number } \\
\%\end{array}$ & $\begin{array}{l}\text { Number } \\
\%\end{array}$ \\
\hline \multirow{2}{*}{$\begin{array}{l}\text { Attended for } \\
\text { asthma review }\end{array}$} & 118 & 427 & 545 \\
\hline & $25.8 \%$ & $31.1 \%$ & $29.7 \%$ \\
\hline \multirow{2}{*}{$\begin{array}{l}\text { Received influenza } \\
\text { vaccination }\end{array}$} & 105 & 345 & 450 \\
\hline & $22.9 \%$ & $25.1 \%$ & $24.6 \%$ \\
\hline \multirow{2}{*}{$\begin{array}{l}\geq 3 \text { issued prescriptions } \\
\text { for an ICS inhaler }\end{array}$} & 161 & 402 & 565 \\
\hline & $35.6 \%$ & $29.3 \%$ & $30.8 \%$ \\
\hline \multirow{2}{*}{$\begin{array}{l}\text { Issued prescription for } \\
\text { oral prednisolone }\end{array}$} & 57 & 84 & 141 \\
\hline & $12.4 \%$ & $6.1 \%$ & $7.7 \%$ \\
\hline \multirow{2}{*}{$\begin{array}{l}\text { Existing clinical code } \\
\text { for COPD }\end{array}$} & 33 & 50 & 83 \\
\hline & $7.2 \%$ & $3.6 \%$ & $4.5 \%$ \\
\hline
\end{tabular}

$2016^{27}$. Therefore, irrespective of the interpretation of the differences between those with a history of OUD and their matched counterparts, the rates of ICS prescriptions seen in this study as well as attendance at asthma review are worryingly low. In a systematic review of strategies for implementing supported self-management for people with asthma ${ }^{11}$, Pinnock and colleagues found that that a whole system approach, targeting patients, professionals and organisations was crucial to improving global asthma care outcomes and that effective interventions were characterised by multi-faceted proactive care. These authors also call for further research into how whole system approaches can be integrated into the routine care of people with asthma and comorbidities as well demographically diverse groups ${ }^{11}$.

There is consensus that the definition of asthma exacerbation should include the systemic use of oral corticosteroids (OCS $)^{30}$ and prescriptions for oral prednisolone have previously been used as a measure of exacerbation from database studies ${ }^{27,31}$. The present study findings, suggest that OUD is associated with increased rates of asthma exacerbations. In addition to the acute risks, asthma exacerbations are independent predictors of future exacerbation $^{32}$ and are correlated with the irreversible airflow limitations over time ${ }^{33}$. There is increasing evidence that inhalation of opioids results in airways disease and in a recent screening study, Burhan et al. ${ }^{34}$ found that most heroin smokers screened in a community setting showed evidence of spirometryconfirmed COPD or COPD-asthma overlap. The present study complements this work as it demonstrates that asthma patients with a history of opioid use have greater rates of COPD independent of some of the main risk factors for the development of this condition, namely tobacco smoking history, deprivation and age $^{35}$. Concurrent diagnoses of asthma and COPD are associated with higher all-cause mortality ${ }^{36}$ and our results suggest that asthma patients with a history of OUD are almost twice as likely to have a comorbid diagnosis of COPD. The odds ratio in our multivariable (adjusted) analysis for the presence of a COPD diagnosis was lower than in the univariate analysis. One tentative interpretation of this is that reducing exacerbations and/ or improving engagement with self-supported care could influence the development of COPD. Further research could explore this possibility by replicating this work in alternative primary care databases.

Clinical recording systems such as ResearchOne and similar databases are widely used and validated for asthma epidemiological research ${ }^{37}$. However, it is known that substance use is underrecorded in primary care databases ${ }^{38}$. In the present study, only $0.2 \%$ of those with a history of asthma were identified as also having a clinical code associated with OUD. The decisions taken by primary care physicians to record substance use are complex ${ }^{39}$ and therefore it is necessary to be cautious in generalising the present results. As this was a database study, it was also not possible to determine current OUD and the findings therefore relate to a lifetime history which may include both current and past OUD. We chose not to omit those who did not have evidence

Table 3. Crude and adjusted odds ratios and $95 \% \mathrm{Cls}$ from conditional logistic regression analysis.

\begin{tabular}{|c|c|c|c|c|}
\hline Outcome (previous 12 months) & Crude OR $(95 \% \mathrm{Cl})$ & $P$-value ${ }^{\mathrm{a}}$ & Adjusted $^{\mathrm{b}}$ OR $(95 \% \mathrm{Cl})$ & $P$-value \\
\hline Attended for asthma review & $0.756(0.591-0.968)$ & 0.026 & $0.600(0.449-0.801)$ & 0.001 \\
\hline Received influenza immunisation & $0.878(0.677-1.139)$ & 0.328 & $0.796(0.594-1.067)$ & 0.127 \\
\hline Issued prescription for oral prednisolone & 1.779 (1.337-2.367) & $<0.001$ & $1.708(1.247-2.339)$ & 0.001 \\
\hline Existing clinical code for COPD & $2.226(1.371-3.613)$ & 0.001 & $1.863(1.120-3.098)$ & 0.017 \\
\hline
\end{tabular}


of active asthma, such as prescription issues within an arbitrary timeframe and did not examine exception reporting (a process by which a patient may be 'excepted' from the targets set in a particular clinical area of the QOF on certain grounds or informed dissent). This was done in an attempt to avoid a potential source of selection bias. There are several potential undesirable consequences of this. Firstly, it is possible that the overall low rates of measured outcomes were simply due to some patients having resolved asthma but with their Read Code not appropriately removed. Secondly, patients may have poorer engagement in part because they have been exception reported. This could potentially explain some of the differences seen between the cases and controls from this study. With respect to ICS use, although our method was pre-specified, a more formal measure of adherence such as the Annual Prescription Possession method may have been desirable to aid comparison with existing literature. Some qualification is also required with respect to matching. As only a basic measure of tobacco smoking history was used (absence or presence) it possible that those with a history of OUD were heavier smokers than those without such histories. More generally, as this is an observational study, there may have been detrimental exposures in the OUD group which were not measured.

Taken together, the results of this study suggest that people with OUD have poor access to asthma care/self-management and worse outcomes on several important measures, despite controlling for important factors which are known to have deleterious effects on asthma, such as tobacco smoking and lower socioeconomic status. Those with a history of OUD encounter a complex range of negative determinants of health at a patient, provider and health system level in common with patients from other populations such as those with severe mental health problems. Further research is required to identify reasons for poor asthma care amongst those from vulnerable and marginalized populations at a whole system level and to better understand how OUD affects long-term respiratory outcomes.

\section{METHODS}

Data source

Data came from the ResearchOne clinical research database ${ }^{40}$, which contains pseudo-anonymised pooled data from participating General Practices in England, UK which use the System One clinical recording system. The database contains information from the patient's electronic health record including coded clinical entries called Read codes (the hierarchical clinical terminology system used by UK General Practices ${ }^{41}$ ) and prescribing data. Research ethics approval was granted from North West-Greater Manchester East Research Ethics committee on 12 January 2016 (16/NW/0037). Data were requested in the form of Read codes and British National Formulary (BNF) chapter heading codes. The observation period was the 12 months prior to the 8 October 2016.

\section{Study design, population and outcome measures}

A matched case-control study was conducted to examine differences between asthma patients with and without a history of OUD. The study population consisted of patients in ResearchOne-linked practices with specific Read codes indicating a possible diagnosis of asthma. A Read code list for this purpose (shown in Supplementary Table 1) was compiled following consultation with a public health specialist. This method has previously been shown to have a high positive predictive value for the diagnosis of asthma ${ }^{42}$. Patients were eligible for inclusion if they were aged 16-65 years. Individuals with a history of OUD (cases) were identified using the Read code list shown in Supplementary Table 2. As it is not possible to ascertain current vs. past OUD (or OUD in remission), 'history' here refers to lifetime history of OUD.

Variables of interest were selected to assess important aspects of asthma care and outcomes. Asthma care measures were based on the British Thoracic Society and Scottish Intercollegiate Guidelines Network (BTS/ SIGN) British Guidelines on the management of asthma ${ }^{43}$. These were: attendance at annual asthma review; receiving an influenza vaccination and being issued three or more prescriptions for a corticosteroid (ICS)containing inhaler as preventer therapy. The cut-off of three ICS-containing inhalers was selected as providing the minimum number of inhalers in order to achieve an approximate $80 \%$ adherence for a typical 200-metered dose inhaler at two puffs per day. Annual asthma reviews, in the UK, are structured consultations between a patient and, usually, a nurse, with the aim of assessing current control of symptoms and important aspects of self-management. These are recommended in the UK national guidelines and are a NICE Quality and Outcomes Framework indicator?

In addition, as a proxy measure of acute asthma exacerbation, we examined whether any prescriptions of oral prednisolone had been issued over the 12-month period. In order to minimise the chances of selecting prednisolone prescribed for alternative indications such as rheumatological conditions, we selected only $5 \mathrm{mg}$ tablet issues with quantities which would correspond to a 5 to 7 -day course of $30-40 \mathrm{mg}$. There is increasing recognition of overlap between asthma and chronic obstructive pulmonary disease $(\mathrm{COPD})^{44}$. Progression of asthma to COPD may be driven, in part, by exacerbations ${ }^{45}$ and there is evidence of an association between heroin inhalation and the early development of emphysema ${ }^{46}$. We, therefore, sought to explore this further by including comorbid diagnoses of COPD. Comorbid diagnoses of COPD were identified through the selection of appropriate Read codes shown in Supplementary Table 3.

\section{Matching}

Cases and controls were matched 1:3 on four confounding variables: age (within 3 years), gender, any recorded smoking history and socioeconomic status (SES). Multiple controls allow for increased statistical power and whilst there is a general consensus that increasing the case-to-control ratio above 1:4 results in very little gain in efficiency, some authors have argued for greater ratios under certain circumstances ${ }^{47}$. The trade-off for this however is that there may be a reduction in the overall matched sample size. In the present study, a 1:3 ratio resulted in the loss of only one case and was therefore chosen as the best compromise between statistical efficiency and matched sample size. The English 2010 Index of Multiple Deprivation (IMD) was used as the SES measure for this study. The IMD contains 38 indicators, organised across seven domains of deprivation which are combined to calculate an overall score representing the degree of deprivation experienced by people living in an area ${ }^{48}$. The IMD score associated with the patient's last known address during the observation period was used and converted to deciles for the purpose of matching.

\section{Statistical analyses}

Differences between asthma patients with and without a history of OUD with respect to the matching variables were made for illustrative purposes. Proportions were assessed using chi-squared tests and mean differences in age were assessed with $95 \%$ confidence intervals and a two-tailed independent group's t-test. Adjusted and unadjusted (crude) odds ratios and $95 \%$ confidence intervals were calculated using conditional logistic regression ${ }^{49}$. All analyses and data handling were conducted using SPSS version 24. Matching was conducted using the SPSS Python Essentials case-control matching module.

\section{Reporting summary}

Further information on research design is available in the Nature Research Reporting Summary linked to this article.

\section{DATA AVAILABILITY}

The data that support the findings of this study are available from ResearchOne but restrictions apply to the availability of these data, which were used under license for the current study, and so are not publicly available. Data are, however, available from the authors upon reasonable request and with permission of ResearchOne. The approach taken for the study is detailed in the main text and, and could be reproduced in any similarly structured linked database.

\section{CODE AVAILABILITY}

No custom software was developed. Only standard use of existing routines within software for data manipulation and statistical analysis was employed. 
Received: 13 June 2019; Accepted: 5 March 2020;

Published online: 20 April 2020

\section{REFERENCES}

1. NHS Digital. Quality and Outcomes Framework (QOF), England: 2017-18: Prevalence, Acheivement and Exceptions Report (2018).

2. Lim, S. F. et al. Frequent attenders to the ED: patients who present with repeated asthma exacerbations. Am. J. Emerg. Med. 32, 895-899 (2014).

3. Caponnetto, P. et al. "Dangerous Relationships": asthma and substance abuse. J. Addict. Dis. 32, 158-167 (2013).

4. Rome, L. A., Lippmann, M. L., Dalsey, W. C., Taggart, P. \& Pomerantz, S. Prevalence of cocaine use and its impact on asthma exacerbation in an urban population. Chest 117, 1324-1329 (2000).

5. Doshi, V. et al. Profile of acute asthma exacerbation in drug users. Am. J. Ther. 24, e39-e43 (2017).

6. Levine, M., Iliescu, M. E., Margellos-Anast, H., Estarziau, M. \& Ansell, D. A. The effects of cocaine and heroin use on intubation rates and hospital utilization in patients with acute asthma exacerbations. Chest 128, 1951-1957 (2005).

7. Eurostat. Causes of death-deaths by country of residence and occurrence. http://appsso.eurostat.ec.europa.eu/nui/show.do?dataset=hlth_cd_aro\&lang=en (2019).

8. Royal College of Physicians. Why asthma still kills, The National Review of Asthma Deaths (NRAD): Confidential Enquiry Report May 2014 (Royal College of Physicians, London, 2014).

9. Williams, L. K. et al. Quantifying the proportion of severe asthma exacerbations attributable to inhaled corticosteroid nonadherence. J. Allergy Clin. Immun. 128, 1185-U1500 (2011)

10. Gibson, P. G. et al. Self-management education and regular practitioner review for adults with asthma. Cochrane Database Syst. Rev. CD001117. https://doi.org/ 10.1002/14651858.CD001117(2002).

11. Pinnock, H. et al. Implementing supported self-management for asthma: a systematic review and suggested hierarchy of evidence of implementation studies. BMC Med. 13, 127 (2015).

12. Vasileiou, E. et al. Effectiveness of influenza vaccines in asthma: a systematic review and meta-analysis. Clin. Infect. Dis. 65, 1388-1395 (2017).

13. Ross, L. E. et al. Barriers and facilitators to primary care for people with mental health and/or substance use issues: a qualitative study. BMC Fam. Pract. 16, 135 (2015).

14. Stover, H. J. \& Schaffer, D. SMOKE IT! Promoting a change of opiate consumption pattern - from injecting to inhaling. Harm Reduct. J. 11, 18 (2014).

15. Strang, J., Griffiths, P. \& Gossop, M. Heroin smoking by 'chasing the dragon': origins and history. Addiction 92, 673-683 (1997).

16. Withington, D. E., Patrick, J. A. \& Reynolds, F. Histamine-release by morphine and diamorphine in man. Anaesthesia 48, 26-29 (1993).

17. Underner, M., Perriot, J., Peiffer, G. \& Jaafari, N. Asthma and heroin use. Presse Med. 46, 660-675 (2017).

18. Tashkin, D. P. Heroin smoking and COPD a case for targeted screening spirometry. Chest 155, 247-248 (2019).

19. Zirakzadeh, A., Shuman, C., Stauter, E., Hays, J. T. \& Ebbert, J. O. Cigarette smoking in methadone maintained patients: an up-to-date review. Curr. Drug Abus. Rev. 6 77-84 (2013)

20. Hurt, R. D. et al. Mortality following inpatient addictions treatment-role of tobacco use in a community-based cohort. JAMA 275, 1097-1103 (1996).

21. Blakey, J. D. et al. Identifying risk of future asthma attacks using uk medical record data: a Respiratory Effectiveness Group initiative. J. Allergy Clin. Immun. Pract. 5 1015 (2017).

22. Bloom, C. I. et al. Exacerbation risk and characterisation of the UK's asthma population from infants to old age. Thorax 73, 313-320 (2018).

23. Yang, J. F. et al. Insights into frequent asthma exacerbations from a primary care perspective and the implications of UK National Review of Asthma Deaths recommendations. Npj Prim. Care Respir. Med. 28, 35 (2018).

24. Al Sallakh, M. A., Rodgers, S. E., Lyons, R. A., Sheikh, A. \& Davies, G. A. Socioeconomic deprivation and inequalities in asthma care in Wales. Lancet 390 S19-S19 (2017).

25. FitzGerald, J. M. \& Gibson, P. G. Asthma exacerbations. 4: prevention. Thorax 61 992-999 (2006)

26. NHS England. Enhanced services specification in Seasonal influenza and pneumococcal immunisation enhanced service, NHS England gateway reference: 01790 (NHS England, 2019).
27. Bloom, C. I., Saglani, S., Feary, J. et al. Changing prevalence of current asthma and inhaled corticosteroid treatment in the UK: population-based cohort 2006-2016. Eur. Respir. J. 53. https://doi.org/10.1183/13993003.02130-2018 (2019).

28. Azar, P. et al. Drug use patterns associated with risk of non-adherence to antiretroviral therapy among HIV-positive illicit drug users in a Canadian setting: a longitudinal analysis. BMC Infect. Dis. 15, 193 (2015).

29. Barnes, C. B. \& Ulrik, C. S. Asthma and adherence to inhaled corticosteroids: current status and future perspectives. Respir. Care 60, 455-468 (2015).

30. Fuhlbrigge, A. L. et al. The burden of asthma in the United States: level and distribution are dependent on interpretation of the national asthma education and prevention program guidelines. Am. J. Respir. Crit. Care Med. 166, 1044-1049 (2002).

31. Suruki, R. Y., Daugherty, J. B., Boudiaf, N. \& Albers, F. C. The frequency of asthma exacerbations and healthcare utilization in patients with asthma from the UK and USA. Bmc Pulm. Med. 17, 74 (2017).

32. Miller, M. K., Lee, J. H., Miller, D. P., Wenzel, S. E. \& Group, T. S. Recent asthma exacerbations: a key predictor of future exacerbations. Respir. Med. 101, 481-489 (2007).

33. Matsunaga, K. et al. Progression of irreversible airflow limitation in asthma: correlation with severe exacerbations. J. Allergy Clin. Immunol. Pract. 3, 759-764 e751 (2015).

34. Burhan, $\mathrm{H}$. et al. Screening heroin smokers attending community drug services for COPD. Chest 155, 279-287 (2019).

35. Antuni, J. D. \& Barnes, P. J. Evaluation of individuals at risk for COPD: beyond the scope of the global initiative for chronic obstructive lung disease. Chron. Obstr. Pulm. Dis. 3, 653-667 (2016).

36. Kendzerska, T. et al. Concurrent physician-diagnosed asthma and chronic obstructive pulmonary disease: a population study of prevalence, incidence and mortality. Plos One 12, e0173830 (2017).

37. Nissen, F. et al. Validation of asthma recording in electronic health records: protocol for a systematic review. Bmj Open 7, e014694 (2017).

38. Davies H. R. Drug use and opioid substitution treatment in pregnancy: evidence from electronic health records. Doctoral thesis, UCL, London (2017).

39. Davies-Kershaw, H., Petersen, I., Nazareth, I. \& Stevenson, F. Factors influencing recording of drug misuse in primary care: a qualitative study of GPs in England. Brit J. Gen. Pract. 68, E234-E244 (2018).

40. ResearchOne. ResearchOne Database System Summary. http://www.researchone org/wp-content/uploads/2013/09/ResearchOne-Database-System-Summary_V2. pdf (2013).

41. Stuart-Buttle, C. D., Read, J. D., Sanderson, H. F. \& Sutton, Y. M. A language of health in action: Read Codes, classifications and groupings. Proceedings: a conference of the American Medical Informatics Association. AMIA Fall Symposium, $75-79$ (1996).

42. Nissen, F. et al. Validation of asthma recording in the Clinical Practice Research Datalink (CPRD). Bmj Open 7, e017474 (2017).

43. Scottish Intercollegiate Guidelines Network and British Thoracic Society. British guideline on the management of asthma: A national clinical guideline, 153 (Scottish Intercollegiate Guidelines Network (SIGN), 2016).

44. Gibson, P. G. \& McDonald, V. M. Asthma-COPD overlap 2015: now we are six. Thorax 70, 683-691 (2015)

45. Gibson, P. G. \& Simpson, J. L. The overlap syndrome of asthma and COPD: what are its features and how important is it? Thorax 64, 728-735 (2009).

46. Walker, P. P., Thwaite, E., Amin, S., Curtis, J. M. \& Calverley, P. M. A. The association between heroin inhalation and early onset emphysema. Chest 148, 1156-1163 (2015).

47. Hennessy, S., Bilker, W. B., Berlin, J. A. \& Strom, B. L. Factors influencing the optimal control-to-case ratio in matched case-control studies. Am. J. Epidemiol. 149, 195-197 (1999).

48. Noble, M., Wright, G., Smith, G. \& Dibben, C. Measuring multiple deprivation at the small-area level. Environ. Plan. A 38, 169-185 (2006).

49. Breslow, N. E., Day, N. E., Halvorsen, K. T., Prentice, R. L. \& Sabai, C. Estimation of multiple relative risk functions in matched case-control studies. Am. J. Epidemiol. 108, 299-307 (1978)

\section{ACKNOWLEDGEMENTS}

This research was funded by the National Institute for Health Research (NIHR) Collaborations for Leadership in Applied Health Research and Care (CLAHRC) with sponsorship from the Academic Health Science network. P.O. is a NIHR/HEE clinical lecturer. We are grateful to Professor Chris Burton for his comments and suggestions on drafts of this paper and to the reviewers for their helpful contributions. We would also like to thank Dr Emily Wood, Dr David Saxon and John Soady for supporting the development of the study protocol. 


\section{AUTHOR CONTRIBUTIONS}

P.O., J.H. and C.M. contributed substantially to the study design, interpretation of results and writing of the paper. P.O. conducted the data analysis and led on preparation of the paper.

\section{COMPETING INTERESTS}

The authors declare no competing interests.

\section{ADDITIONAL INFORMATION}

Supplementary information is available for this paper at https://doi.org/10.1038/ s41533-020-0174-2.

Correspondence and requests for materials should be addressed to P.O.

Reprints and permission information is available at http://www.nature.com/ reprints
Publisher's note Springer Nature remains neutral with regard to jurisdictional claims in published maps and institutional affiliations.

Open Access This article is licensed under a Creative Commons adaptation, distribution and reproduction in any medium or format, as long as you give appropriate credit to the original author(s) and the source, provide a link to the Creative Commons license, and indicate if changes were made. The images or other third party material in this article are included in the article's Creative Commons license, unless indicated otherwise in a credit line to the material. If material is not included in the article's Creative Commons license and your intended use is not permitted by statutory regulation or exceeds the permitted use, you will need to obtain permission directly from the copyright holder. To view a copy of this license, visit http://creativecommons. org/licenses/by/4.0/.

(c) The Author(s) 2020 